\title{
Effects of candidate gene polymorphisms on the detailed fatty acids profile determined by gas chromatography in bovine milk
}

\author{
S. Pegolo, ${ }^{*}$ A. Cecchinato, ${ }^{* 1}$ M. Mele, $†$ G. Conte, $\dagger$ S. Schiavon, ${ }^{*}$ and G. Bittante* \\ *Department of Agronomy, Food, Natural Resources, Animals and Environment (DAFNAE), University of Padova, Viale dell'Università 16, \\ 35020 Legnaro, Padova, Italy \\ †Department of Agriculture, Food and Environment, Università di Pisa, Via del Borghetto, 80, 56124 Pisa, Italy
}

\begin{abstract}
Association analyses between candidate genes and bovine milk fatty acids can improve our understanding of genetic variation in milk fatty acid profiles and reveal potential opportunities to tailor milk fat composition through selection strategies. In this work, we investigated the association of 51 single nucleotide polymorphisms (SNP) selected from 37 candidate genes using a functional and positional approach, with 47 fatty acids, 9 fatty acid groups, and $5 \Delta^{9}$-desaturation indices in milk samples from Brown Swiss cows. Individual milk samples were collected from 1,158 Italian Brown Swiss cows, and gas chromatography was used to obtain detailed milk fatty acid compositions. A GoldenGate assay system (Illumina, San Diego, CA) was used to perform genotype 96 selected SNP located in 54 genes across 22 chromosomes. In total, 51 polymorphic SNP in 37 candidate genes were retained for the association analysis. A Bayesian linear animal model was used to estimate the contribution of each SNP. A total of 129 tests indicated relevant additive effects between a given SNP and a single fatty acid trait; 38 SNP belonging to 30 genes were relevant for a total of 57 fatty acid traits. Most of the studied fatty acid traits $(\sim 81 \%)$ were relevantly associated with multiple SNP. Relevantly associated SNP were mainly found in genes related to fat metabolism, linked to or contained in previously identified quantitative trait loci for fat yield or content, or associated with genes previously identified in association analyses with milk fatty acid profiles in other cow breeds. The most representative candidate genes were $L E P, P R L, S T A T 5 A, C C L 3$, ACACA, GHR, ADRB2, LPIN1, STAT1, FABP4, and CSN2. In particular, relevant associations with SNP located on bovine chromosome 19 (BTA19) were found.
\end{abstract}

Received September 18, 2015.

Accepted February 10, 2016.

${ }^{1}$ Corresponding author: alessio.cecchinato@unipd.it
Two candidate genes on BTA19 (CCL3 and ACACA) were relevantly associated with de novo short- and medium-chain fatty acids, likely explaining the high heritability values found for these fatty acids (with the exception of C6:0). Two additional genes on BTA19 (CCL2 and GH1) showed associations with saturated and branched-chain fatty acids. Our findings provide basic information on genes and SNP affecting the milk fatty acid composition of dairy cows. These results may support the possibility of using genetic selection to modify milk fatty acid profiles to promote beneficial health-related effects.

Key words: association analyses, candidate genes, milk fatty acids, single nucleotide polymorphism

\section{INTRODUCTION}

Dairy products contain a wide range of saturated and unsaturated fatty acids with chain lengths ranging from 4 to $>20$ carbon atoms. Regulation of the fatty acid composition of milk has received attention because certain fatty acids are believed to have various beneficial or potentially harmful effects on human health. However, this issue is still very controversial, with recent studies seeming to contradict the traditional accepted role of specific fatty acids in health and disease. For instance, recent human meta-analyses found no association between SFA intake and the risk of cardiovascular disease or stroke (Mente et al., 2009; Siri-Tarino et al., 2010). Some epidemiological studies have suggested that coronary heart disease risk may be positively associated with industrial trans fatty acids but not those originating from biohydrogenation in ruminants, including vaccenic acid (VA) and the naturally occurring isomer of CLA or C18:2 cis-9,trans-11 (rumenic acid, RA; Gebauer et al., 2011). The biologically beneficial effects of CLA have been widely documented (Dilzer and Park, 2012), and both n-6 and n-3 PUFA are shown to reduce cardiovascular risk (Michas et al., 2014). However, the benefits of n-3 fatty acids [e.g., eicosapentaenoic acid (EPA) and docosahexaenoic acid (DHA)] seem to be not as pronounced as previously believed, warranting 
caution when recommending dietary supplementation of PUFA to the general population without considering the individual intake of total energy and fats.

Because of this increasing awareness of the relationships between diet and health, several feeding and genetic strategies have been evaluated for their ability to engineer milk fat toward a healthier composition (Mele, 2009; Shingfield et al., 2013). In a few European countries (e.g., France and the Netherlands), the dairy industry recently introduced supplementary premiums for farmers based on milk fatty acid compositions (e.g., SFA, n-3 fatty acids, and total C18:1 fatty acids), and contents of $\alpha$-linolenic acid (C18:3 cis-9, cis-12,cis-15), oleic acid (C18:1 cis-9), and palmitic acid (C16:0; Borreani et al., 2013). Feeding trials have clearly demonstrated that altering the cow's nutrition is a very effective way to quickly change the milk fat composition; however, these effects are completely reversible when feeding conditions change and the necessary changes can significantly increase the feeding cost (Shingfield et al., 2013).

In contrast, genetic improvement can gradually induce smaller but permanent trait modifications. Genetic analyses have shown heritable variations in the fatty acid profile of bovine milk (e.g., Bastin et al., 2013; Krag et al., 2013; Pegolo et al., 2016). Moreover, candidate gene studies have shown that SNP in the genes encoding stearoyl-CoA desaturase (SCD), diacylglycerol o-acyltransferase 1 (DGAT1), and sterol regulatory element binding protein-1 (SREBP1) clearly influence the milk fat composition and explain part of the genetic variation of milk fat unsaturation indices seen in Holsteins (Schennink et al., 2007, 2008; Rincon et al., 2012) and Brown Swiss cows (Mele et al., 2007; Conte et al., 2010). Single nucleotide polymorphisms located in other genes involved in lipid biosynthesis have also been shown to affect the milk fatty acid compositions of different bovine breeds (Matsumoto et al., 2012; Marchitelli et al., 2013; Nafikov et al., 2014). Genomewide studies in Holstein cows have allowed researchers to detect QTL harboring candidate polymorphic genes that are involved in fat synthesis or known to affect fat yield or content, and significantly associated with fatty acids ( $\sim 20$ fatty acid traits were considered) (e.g., Stoop et al., 2009; Bouwman et al., 2011). Recently, a selective DNA-pooling approach was used to identify QTL for milk content of RA, VA, and SCD (Strillacci et al., 2014).

These results collectively suggest that loci found to affect fatty acid profiles could be used in markerassisted selection programs aimed at modifying the nutritional qualities of milk. However, relatively few fatty acids have been examined to date in this context, and several interesting fatty acids are not yet well known, especially those present at relatively low amounts in milk (and thus hard to detect) but that could have a role in human health.

This work is part of a broader project including the evaluation of the effects of polymorphisms in candidate genes on production traits, milk technological properties, and milk fatty acid profile in Brown Swiss cows. We previously analyzed 96 SNP in 54 candidate genes, and 51 polymorphic SNP in 37 candidate genes were retained for association analyses with productivity and technological traits (Cecchinato et al., 2014, 2015). Here, we extend our association analysis to a very detailed milk fatty acid profile, including 47 individual fatty acids, 9 fatty acid groups, and $5 \Delta^{9}$-desaturation indices, determined by $\mathrm{GC}$ in the same population. Our aim is to develop genetic markers that will allow breeders to select for animals that produce milk with a healthier fatty acid composition.

\section{MATERIALS AND METHODS}

\section{Animals and Milk Sampling}

The milk sampling procedure is detailed in CipolatGotet et al. (2013). Briefly, milk samples were collected once per cow during the evening milking from 1,158 Brown Swiss cows from 85 herds (a maximum of 15 cows per herd) located in the Alpine province of Trento (Italy). Each farm was sampled once. The milk samples (without the addition of preservative) were immediately refrigerated at $4^{\circ} \mathrm{C}$ and transferred to the Cheese-Making Laboratory of the Department of Agronomy, Food, Natural Resources, Animals and Environment of the University of Padova (Legnaro, Padova, Italy). Data regarding the cows and herds were provided by the $\mathrm{Su}$ perbrown Consortium of Bolzano and Trento (Italy), and pedigree information was obtained from the Italian Brown Swiss Cattle Breeders Association (ANARB, Verona, Italy). Each sampled cow had known ancestors for at least 4 generations and the pedigree file included 8,845 animals; there were 1,326 sires, 264 of which had progeny (between 2 and 80 daughters) with records in the data set.

\section{GC Analysis}

Fatty acid methyl esters were prepared by the direct extraction and alkali-catalyzed trans-methylation procedure previously described by Feng et al. (2004) and modified as in Pegolo et al. (2016). The fatty acid composition was determined using a ThermoQuest gas chromatograph (ThermoElectron Corp., Waltham, MA) equipped with a flame-ionization detector and a high polar fused-silica capillary column (Chrompack 
CP-Sil88 Varian, Middelburg, the Netherlands; 100 $\mathrm{m}, 0.25 \mathrm{~mm}$ i.d.; film thickness $0.20 \mu \mathrm{m}$ ). Individual FAME were identified by comparison with standard mixtures, pure standards, and published GC profiles. In the case of $\mathrm{C} 16: 1, \mathrm{C} 18: 1$, and $\mathrm{C} 18: 2$ isomers, risk of peak coelution was assessed by using an Ag-ion solidphase extraction fractionation, according to Kramer et al. (2008). A reference standard butter (BCR 164; Commission of the European Communities, Community Bureau of Reference, Brussels, Belgium) was used to estimate correction factors for the short-chain fatty acids (SCFA), as previously described by Mele et al. (2008). Inter- and intraassay coefficients of variation were calculated using the reference standard butter, and the detection limit of the analysis was $0.001 \%$ above that of the total fatty acids amount. The milk fatty acid composition was expressed as grams per 100 $\mathrm{g}$ of total fatty acids.

\section{Blood Sampling, DNA Extraction, SNP Selection, and Genotyping}

Blood sampling, DNA extraction, and SNP selection and genotyping were performed as previously described (Cecchinato et al., 2014). Briefly, DNA extraction was performed with a DNeasy 96 Blood and Tissue Kit (Qiagen, Hilden, Germany) from $100 \mu \mathrm{L}$ of individual whole blood. The quantity and quality of the obtained DNA were assessed using the QBit system (Invitrogen, Carlsbad, CA) and 1\% agarose gel electrophoresis. Candidate gene selection was carried out using both a functional candidate gene approach (genes involved in the synthesis of proteins, fatty acids, and components of the immune system) and a positional candidate gene approach (location in chromosomal regions associated with milk quality and technological properties). From a total of 113 SNP initially selected, the 89 SNP with the best Illumina designability rank scores, and 7 SNP with scores between 0.5 and 0.6 were chosen. The GoldenGate system (Illumina, San Diego, CA) was used to genotype the 96 selected SNP, which were located in 54 genes on 22 chromosomes. Fifty-one polymorphic SNP [minor allele frequency (MAF) $>0.05]$ in 37 candidate genes were retained for the association analysis. Details on all of the genotyped SNP (e.g., chromosomal location, genomic position, type of mutation, MAF) are reported in the Supplemental Data file (http://dx.doi. org/10.3168/jds.2015-10420).

\section{Statistical Analysis}

Association studies for all investigated genes were carried out using Bayesian methodology (Sorensen and Gianola, 2002). The following mixed linear animal model was used:

$$
\mathrm{y}_{i j k l}=\mu+\mathrm{DIM}_{i}+\text { parity }_{j}+\mathrm{h}_{k}+\mathrm{a}_{l}+\mathrm{x}_{l m} \beta_{m}+\varepsilon_{i j k l},
$$

where $\mathrm{y}_{i j k l}$ is the phenotypic record for the analyzed trait; $\mathrm{DIM}_{i}$ is the effect of the ith class of days in milk ( $i=1$ to 6 ; class $1,<60 \mathrm{~d}$; class 2, 60 to $120 \mathrm{~d}$; class 3 , 121 to $180 \mathrm{~d}$; class 4,181 to $240 \mathrm{~d}$; class 5,241 to 300 $\mathrm{d}$; and class $6,>300 \mathrm{~d}$ ); parity $_{j}$ is the effect of the $j$ th parity of the cow ( $j=1$ to 5 or more); $\mathrm{h}_{k}$ is the effect of the $k$ th herd ( $k=1$ to 85$) ; \mathrm{a}_{l}$ is the polygenic effect of individual $l ; \mathrm{x}_{l m}(0,1,2)$ reflects the number of copies of the minor allele at the $m$ th SNP of subject $l ; \beta_{m}$ is the additive effect of the $m$ th SNP; and $\varepsilon_{i j k l}$ is the random residual term.

Bounded uniform priors were used for all environmental variables, and $\mathbf{a}$ and $\mathbf{h}$ were assumed a priori to be independent and normally distributed:

$$
\begin{aligned}
& \mathbf{a} \mid \sigma_{a}^{2} \sim N\left(\mathbf{0}, \mathbf{A} \sigma_{a}^{2}\right), \\
& \mathbf{h} \mid \sigma_{h}^{2} \sim N\left(\mathbf{0}, \mathbf{I} \sigma_{h}^{2}\right),
\end{aligned}
$$

where $\mathbf{A}$ is the known additive genetic relationship matrix, $\mathbf{I}$ is the identity matrix, and $\sigma_{h}^{2}$ and $\sigma_{a}^{2}$ were herd and additive genetic variances, respectively. The pedigree file included information on 8,845 animals; there were 1,326 sires, 264 of which had progeny (between 2 and 80 daughters) with records in the data set.

The marginal posterior distributions of all parameters were obtained using a Gibbs sampler running with a single chain of 1,000,000 points; the first 50,000 were discarded as burn-in, as previously tested by Raftery and Lewis (1996). Samples were saved every 100 iterations. Due to autocorrelations between successive samples, convergence was tested using Geweke's Zcriterion (Geweke, 1992). Monte Carlo sampling errors and the effective sample size were computed using the time-series procedures described by Geyer (1992). The parameters of concern were the dispersion parameters and the additive effects of SNP, as defined by Falconer and Mackay (1996). The posterior mean was used as a point estimate for the parameter of concern. The lower and upper bounds of the 95\% highest posterior probability density regions (HPD95) for each additive effect were estimated from the Gibbs samples.

For all traits, the model was fitted to separately estimate the contribution of each SNP (i.e., the model was run 51 times per trait for a total of 3,111 times). A SNP was considered as having a relevant effect on the 
Table 1. Descriptive statistics for milk production traits and individual SFA (g/100 $\mathrm{g}$ of fatty acids)

\begin{tabular}{|c|c|c|c|c|c|}
\hline Milk production trait & Mean & $\mathrm{SD}$ & $\mathrm{P} 5^{1}$ & $\mathrm{P} 95^{1}$ & Heritability $^{2}$ \\
\hline Milk yield, kg/d & 24.43 & 7.95 & 12.11 & 37.90 & 0.086 \\
\hline Fat, \% & 4.23 & 0.73 & 3.15 & 5.45 & 0.259 \\
\hline \multicolumn{6}{|l|}{ Individual fatty acids } \\
\hline $\mathrm{C} 4: 0$ & 3.46 & 0.91 & 2.09 & 4.99 & 0.033 \\
\hline C6:0 & 2.15 & 0.39 & 1.50 & 2.79 & 0.039 \\
\hline $\mathrm{C} 8: 0$ & 1.35 & 0.23 & 0.97 & 1.70 & 0.124 \\
\hline C10:0 & 3.17 & 0.63 & 2.15 & 4.17 & 0.201 \\
\hline C11:0 & 0.06 & 0.04 & 0.02 & 0.13 & 0.134 \\
\hline $\mathrm{C} 12: 0$ & 3.72 & 0.75 & 2.47 & 4.86 & 0.243 \\
\hline C13:0 & 0.11 & 0.04 & 0.06 & 0.18 & 0.078 \\
\hline $\mathrm{C} 13: 0$ iso & 0.06 & 0.04 & 0.02 & 0.14 & 0.034 \\
\hline $\mathrm{C} 14: 0$ & 12.08 & 1.56 & 9.36 & 14.15 & 0.097 \\
\hline $\mathrm{C} 14: 0$ iso & 0.17 & 0.05 & 0.08 & 0.26 & 0.125 \\
\hline $\mathrm{C} 15: 0$ & 1.19 & 0.24 & 0.82 & 1.59 & 0.102 \\
\hline $\mathrm{C} 15: 0$ iso & 0.28 & 0.08 & 0.17 & 0.42 & 0.092 \\
\hline C15:0 ante & 0.53 & 0.12 & 0.35 & 0.73 & 0.115 \\
\hline $\mathrm{C} 16: 0$ & 30.54 & 3.72 & 24.67 & 36.67 & 0.255 \\
\hline $\mathrm{C} 16: 0$ iso & 0.32 & 0.09 & 0.18 & 0.47 & 0.077 \\
\hline C17:0 & 0.54 & 0.12 & 0.39 & 0.75 & 0.092 \\
\hline $\mathrm{C} 17: 0$ iso & 0.32 & 0.08 & 0.18 & 0.45 & 0.050 \\
\hline $\mathrm{C} 17: 0$ ante & 0.42 & 0.09 & 0.29 & 0.56 & 0.102 \\
\hline C18:0 & 8.95 & 1.87 & 6.19 & 12.50 & 0.218 \\
\hline C20:0 & 0.13 & 0.04 & 0.07 & 0.20 & 0.097 \\
\hline $\mathrm{C} 22: 0$ & 0.06 & 0.03 & 0.02 & 0.12 & 0.031 \\
\hline C24:0 & 0.04 & 0.02 & 0.01 & 0.08 & 0.031 \\
\hline
\end{tabular}

${ }^{1} \mathrm{P} 5=5$ th percentile; $\mathrm{P} 95=95$ th percentile.

${ }^{2}$ From Pegolo et al. (2016).

trait when the posterior means of the additive effect did not include zero in the HPD95 interval. Moreover, as suggested by Ramírez et al. (2014), we computed the posterior probability of the estimated effect of being $>0$ for positive effects or $<0$ for negative effects (PPNO). Only relevant SNP are presented in the tables. The genetic variance explained by a given $\operatorname{SNP}\left(\mathbf{V}_{\mathbf{a}}\right)$ was calculated from the estimated genotypic effects and the observed genotypic frequencies.

Descriptive statistics and estimates of genetic variance components are given in Tables 1, 2, and 3 and were extensively reported and discussed in Pegolo et al. (2016). The present results are expressed as the percentage of the total additive genetic variance obtained using the same data set.

\section{RESULTS AND DISCUSSION}

\section{Associations of Candidate Genes with Milk Fatty Acid Profile}

Several prior studies have taken the approach of performing association analyses between candidate genes and the milk fatty acid profile, but these studies considered relatively few genes and fatty acid traits (e.g., Rincon et al., 2012; Marchitelli et al., 2013; Nafikov et al., 2014). Moreover, to our knowledge, only one previ- ous study reported association analyses with the milk fatty acid profile of the Brown Swiss breed (Strillacci et al., 2014). In that study, the authors performed genotyping using the Illumina BovineSNP50 BeadChip, but they considered only VA and RA as fatty acid traits.

In the present work, 38 different SNP belonging to 30 genes were relevant for 57 fatty acid traits (Tables 4,5 , and 6 ). The most representative and relevantly associated candidate genes are outlined in Table 7 .

\section{Leptin}

The leptin (LEP) gene, which is located on BTA4, was associated with 9 fatty acids and 2 desaturation indices, giving it the greatest number of associations among the genes considered in the present study (Table 7). The $L E P$ gene encodes a hormone that plays a key role in the metabolic adaptation of nutrient partitioning during the energy-consuming processes of pregnancy and lactation. At the onset of lactation, LEP in the presence of prolactin (PRL) enhances fat synthesis and elevates the expression levels of the genes encoding $\alpha-\mathrm{CN}$ and $\beta$-LG (Feuermann et al., 2004).

We identified relevant associations between fatty acids and 2 SNP of LEP: rs 110559656 and rs29004170. The $A$ allele of rs 110559656 was negatively associated with several short- and medium-chain SFA (from C6:0 
PEGOLO ET AL.

Table 2. Descriptive statistics for individual UFA (g/100 g of fatty acids)

\begin{tabular}{|c|c|c|c|c|c|}
\hline Individual fatty acids & Mean & SD & $\mathrm{P} 5^{1}$ & $\mathrm{P} 95^{1}$ & Heritability $^{2}$ \\
\hline \multicolumn{6}{|l|}{ MUFA } \\
\hline C10:1 cis-9 & 0.33 & 0.09 & 0.18 & 0.47 & 0.124 \\
\hline $\mathrm{C} 14: 1$ cis-9 & 1.08 & 0.32 & 0.54 & 1.61 & 0.360 \\
\hline C16:1 cis-9 & 1.21 & 0.32 & 0.78 & 1.80 & 0.243 \\
\hline C16:1 trans -9 & 0.06 & 0.03 & 0.02 & 0.12 & 0.033 \\
\hline $\mathrm{C} 17: 1$ cis-9 & 0.20 & 0.08 & 0.11 & 0.34 & 0.070 \\
\hline $\mathrm{C} 18: 1$ trans -4 & 0.03 & 0.02 & 0.01 & 0.08 & 0.023 \\
\hline C18:1 trans $-6-8$ & 0.21 & 0.07 & 0.11 & 0.34 & 0.059 \\
\hline C18:1 trans-9 & 0.18 & 0.06 & 0.11 & 0.27 & 0.082 \\
\hline $\mathrm{C} 18: 1$ trans -10 & 0.29 & 0.10 & 0.17 & 0.45 & 0.056 \\
\hline C18:1 trans-11 (vaccenic acid) & 1.20 & 0.38 & 0.61 & 1.85 & 0.074 \\
\hline C18:1 cis-9 & 18.33 & 3.21 & 14.03 & 23.43 & 0.089 \\
\hline C18:1 cis-12 & 0.24 & 0.10 & 0.11 & 0.43 & 0.083 \\
\hline C18:1 trans -16 & 0.24 & 0.08 & 0.14 & 0.40 & 0.058 \\
\hline $\mathrm{C} 20: 1$ cis-9 & 0.11 & 0.04 & 0.05 & 0.17 & 0.064 \\
\hline \multicolumn{6}{|l|}{ PUFA } \\
\hline C18:2 cis- 9, trans-11 (rumenic acid) & 0.65 & 0.22 & 0.32 & 1.03 & 0.072 \\
\hline $\mathrm{C} 18: 2$ trans- 11, cis- 15 & 0.10 & 0.08 & 0.04 & 0.18 & 0.032 \\
\hline C18:2 trans -9, trans -12 & 0.58 & 0.44 & 0.35 & 0.88 & 0.030 \\
\hline $\mathrm{C} 18: 2$ cis- 9, cis- 12 & 2.04 & 0.60 & 1.23 & 3.12 & 0.060 \\
\hline $\mathrm{C} 18: 3$ cis-9,cis-12,cis-15 & 0.56 & 0.17 & 0.30 & 0.86 & 0.093 \\
\hline C18:3 cis-9,trans-11,cis-15 & 0.04 & 0.03 & 0.02 & 0.10 & 0.031 \\
\hline $\mathrm{C} 20: 3$ cis- 8, cis-11,cis-14 & 0.10 & 0.06 & 0.05 & 0.21 & 0.109 \\
\hline $\mathrm{C} 20: 4$ cis- 5, cis- 8, cis- 11, cis- 14 & 0.13 & 0.05 & 0.07 & 0.22 & 0.081 \\
\hline C20:5 cis-5, cis-8, cis-11,cis-14,cis-17 & 0.05 & 0.02 & 0.02 & 0.10 & 0.045 \\
\hline $\mathrm{C} 22: 4$ cis- 7, cis- 10, cis- 13, cis- 16 & 0.03 & 0.02 & 0.01 & 0.08 & 0.048 \\
\hline C22:5 cis-7,cis-10,cis-13,cis-16,cis-19 & 0.08 & 0.03 & 0.04 & 0.14 & 0.039 \\
\hline
\end{tabular}

${ }^{1} \mathrm{P} 5=5$ th percentile; $\mathrm{P} 95=95$ th percentile.

${ }^{2}$ From Pegolo et al. (2016).

to C12:0; explaining from 2.4 to $8.7 \%$ of the genetic variance) and positively associated with C18:1 cis-9 (explaining $9 \%$ of the genetic variance). Interestingly, high heritability values have been previously reported for most of the de novo synthesized fatty acids (e.g., C8:0 to C12:0; Pegolo et al., 2016). Substitution of the $A$ allele for the $G$ allele of rs 110559656 reduces SFA and increases MUFA (Table 6). Furthermore, the $G$ allele

Table 3. Descriptive statistics of groups of fatty acids (g/100 g of fatty acids) and unsaturation indices

\begin{tabular}{|c|c|c|c|c|c|}
\hline Item $^{1}$ & Mean & SD & $\mathrm{P} 5^{2}$ & $\mathrm{P} 95^{2}$ & Heritability $^{3}$ \\
\hline \multicolumn{6}{|l|}{ Group of fatty acids } \\
\hline SFA & 69.63 & 4.11 & 61.98 & 75.50 & 0.147 \\
\hline MUFA & 24.24 & 3.46 & 19.52 & 29.88 & 0.132 \\
\hline PUFA & 3.78 & 0.79 & 2.66 & 5.13 & 0.107 \\
\hline Short-chain fatty acids & 10.52 & 1.72 & 7.68 & 13.25 & 0.054 \\
\hline Medium-chain fatty acids & 52.81 & 5.26 & 43.11 & 60.79 & 0.146 \\
\hline Long-chain fatty acids & 34.38 & 5.14 & 26.94 & 43.45 & 0.126 \\
\hline Branched-chain fatty acids & 2.08 & 0.41 & 1.47 & 2.78 & 0.083 \\
\hline Trans fatty acids & 2.22 & 0.53 & 1.42 & 3.07 & 0.107 \\
\hline Trans fatty acids C18:1 & 2.16 & 0.52 & 1.37 & 3.00 & 0.111 \\
\hline \multicolumn{6}{|l|}{ Unsaturation index, \% } \\
\hline C10:1/(C10:0 + C10:1) & 9.54 & 2.00 & 0.06 & 0.13 & 0.227 \\
\hline $\mathrm{C} 14: 1 /(\mathrm{C} 14: 0+\mathrm{C} 14: 1)$ & 8.16 & 2.04 & 0.05 & 0.12 & 0.438 \\
\hline $\mathrm{C} 16: 1 /(\mathrm{C} 16: 0+\mathrm{C} 16: 1)$ & 3.83 & 0.90 & 0.03 & 0.05 & 0.258 \\
\hline $\mathrm{C} 18: 1 /(\mathrm{C} 18: 0+\mathrm{C} 18: 1)$ & 67.22 & 4.32 & 0.60 & 0.73 & 0.218 \\
\hline $\mathrm{RA} /(\mathrm{RA}+\mathrm{VA})$ & 34.98 & 5.53 & 0.26 & 0.44 & 0.098 \\
\hline
\end{tabular}

${ }^{1}$ Short-chain fatty acids included the C4:0, C6:0, C8:0, and C10:0 fatty acids; medium-chain fatty acids included all linear fatty acids from C11:0 to C16:1; long-chain fatty acids included all linear fatty acids from C17:0 to C24:0; trans fatty acids included all trans fatty acids; trans fatty acids C18:1 included all trans isomers of $\mathrm{C} 18: 1 . \mathrm{RA}=$ rumenic acid; $\mathrm{VA}=$ vaccenic acid

${ }^{2} \mathrm{P} 5=5$ th percentile; $\mathrm{P} 95=95$ th percentile.

${ }^{3}$ From Pegolo et al. (2016). 
Table 4. Estimated marginal posterior densities of additive effect for the relevant SNP on individual SFA

\begin{tabular}{|c|c|c|c|c|c|c|c|}
\hline Trait & Gene $^{1}$ & rs no. $^{2}$ & Allele & Effect $^{3}$ & HPD95 ${ }^{4}$ & $\mathrm{PPN}^{5}$ & $\mathrm{~V}_{\mathrm{a}},{ }^{6} \%$ \\
\hline C4:0 & PPARGC1A & rs44857081 & A vs. C & 0.210 & $0.035 ; 0.374$ & 0.992 & 16.1 \\
\hline \multirow{3}{*}{ C6:0 } & $L E P$ & rs29004170 & C vs. $\mathrm{G}$ & 0.030 & $0.003 ; 0.056$ & 0.985 & 7.4 \\
\hline & $\mathrm{FABP}_{4}$ & rs110757796 & A vs. $G$ & 0.056 & $0.002 ; 0.110$ & 0.979 & 14.0 \\
\hline & $L E P$ & rs110559656 & A vs. G & -0.035 & $-0.068 ;-0.003$ & 0.981 & 8.7 \\
\hline C8:0 & CCL3 & rs109686238 & T vs. $\mathrm{C}$ & 0.041 & $0.020 ; 0.062$ & 0.999 & 13.1 \\
\hline \multirow{2}{*}{ C10:0 } & $A C A C A$ & rs110562092 & A vs. G & -0.061 & $-0.113 ;-0.011$ & 0.991 & 2.4 \\
\hline & $L E P$ & rs110559656 & A vs. $G$ & -0.067 & $-0.128 ;-0.007$ & 0.984 & 2.5 \\
\hline \multirow[t]{2}{*}{ C11:0 } & CCR2 & rs41257559 & T vs. C & -0.004 & $-0.007 ;-0.001$ & 0.989 & 0.7 \\
\hline & STAT5A & rs137182814 & C vs. G & -0.031 & $-0.061 ;-0.001$ & 0.978 & 4.8 \\
\hline \multirow[t]{2}{*}{ C12:0 } & CCL3 & rs109686238 & T vs. C & 0.112 & $0.044 ; 0.179$ & 0.999 & 4.6 \\
\hline & AGPAT6 & rs110445169 & T vs. C & 0.064 & $0.001 ; 0.127$ & 0.975 & 1.5 \\
\hline \multirow[t]{2}{*}{ C14:0 } & $C C L 3$ & rs109686238 & T vs. C & 0.154 & $0.019 ; 0.288$ & 0.987 & 5.2 \\
\hline & CSN2 & rs43703011 & A vs. $\mathrm{C}$ & -0.216 & $-0.426 ;-0.005$ & 0.979 & 7.8 \\
\hline \multirow[t]{3}{*}{ C14:0 iso } & $L E P$ & rs29004170 & $\mathrm{C}$ vs. $\mathrm{G}$ & -0.004 & $-0.008 ;-0.001$ & 0.993 & 0.8 \\
\hline & $P R L$ & rs110684599 & A vs. C & 0.010 & $0.004 ; 0.017$ & 0.999 & 3.8 \\
\hline & $C A R D 15$ & rs43710288 & T vs. A & -0.004 & $-0.008 ;-0.001$ & 0.978 & 0.8 \\
\hline \multirow[t]{3}{*}{ C15:0 } & GHR & rs109136815 & T vs. C & 0.021 & $0.001 ; 0.042$ & 0.977 & 3.1 \\
\hline & LPIN1 & rs136905033 & T vs. C & 0.064 & $0.007 ; 0.120$ & 0.986 & 12.3 \\
\hline & $A D R B 2$ & rs132839139 & A vs. $G$ & -0.100 & $-0.188 ;-0.009$ & 0.986 & 15.8 \\
\hline \multirow[t]{3}{*}{$\mathrm{C} 15: 0$ iso } & GHR & rs109136815 & T vs. C & 0.006 & $0.001 ; 0.012$ & 0.975 & 1.5 \\
\hline & $P R L$ & rs110684599 & A vs. C & 0.015 & $0.008 ; 0.023$ & 0.999 & 8.4 \\
\hline & $X D H$ & rs42890834 & A vs. G & -0.005 & $-0.011 ;-0.001$ & 0.978 & 1.2 \\
\hline C15:0 ante & $C C L 2$ & rs 41255714 & A vs. $G$ & -0.010 & $-0.019 ;-0.002$ & 0.991 & 0.2 \\
\hline C16:0 & ORL1 & rs135588030 & A vs. G & -0.486 & $-0.874 ;-0.091$ & 0.994 & 2.4 \\
\hline $\mathrm{C} 17: 0$ & CCR2 & rs41257559 & T vs. $\mathrm{C}$ & -0.009 & $-0.019 ;-0.001$ & 0.978 & 3.5 \\
\hline \multirow[t]{3}{*}{ C20:0 } & $P R L$ & rs110684599 & A vs. C & 0.006 & $0.001 ; 0.012$ & 0.978 & 1.4 \\
\hline & CSN3 & rs43703015 & T vs. C & 0.007 & $0.001 ; 0.013$ & 0.980 & 1.7 \\
\hline & $A D R B 2$ & rs132839139 & A vs. G & 0.022 & $0.003 ; 0.040$ & 0.989 & 4.6 \\
\hline \multirow[t]{3}{*}{$\mathrm{C} 22: 0$} & $S C D 1$ & rs41255693 & T vs. C & 0.007 & $0.000 ; 0.014$ & 0.974 & 1.3 \\
\hline & $C S N 1 S 1$ & rs43703010 & A vs. $G$ & -0.012 & $-0.020 ;-0.004$ & 0.999 & 2.6 \\
\hline & $A D R B 2$ & rs132839139 & A vs. $G$ & 0.014 & $0.001 ; 0.027$ & 0.983 & 1.9 \\
\hline
\end{tabular}

${ }^{1}$ All gene symbols were defined in Cecchinato et al. (2014).

${ }^{2}$ http://www.ncbi.nlm.nih.gov/snp.

${ }^{3} \mathrm{SNP}$ were considered having a relevant effect on the trait when the posterior mean of the additive effect did not include zero in the HPD interval.

${ }^{4}$ HPD95 = lower and upper bound of the $95 \%$ highest posterior density region.

${ }^{5} \mathrm{PPN} 0=$ the probability of the additive effect to be over or below zero.

${ }^{6} \mathrm{~V}_{\mathrm{a}}=$ proportion (\%) of genetic variance explained by each SNP.

of rs29004170 increases some PUFA, such as RA ( $\mathrm{V}_{\mathrm{a}}$ $=6.5 \%)$ and $\mathrm{C} 18: 2$ trans -11, cis $-15\left(\mathrm{~V}_{\mathrm{a}}=3.1 \%\right)$. In the case of the 2 desaturase indices, the SNP had small but relevant effects $\left(\mathrm{V}_{\mathrm{a}}<0.1 \%\right.$; Tables 6 and 7$)$.
Several polymorphisms in $L E P$ have been studied for their associations with milk production traits in dairy cattle (e.g., Buchanan et al., 2003; Clempson et al., 2011; Zetouni et al., 2013). However, contradictory re- 
sults have been found, probably reflecting the presence of population-specific haplotypes or the major role of other genes and QTL in milk production. In previous studies (Cecchinato et al., 2014, 2015), our group did not find any relevant association of SNP on $L E P$ with milk technological or production traits. In their study on genetic variation of milk fatty acids, Marchitelli and colleagues (2013) found no relevant association between

Table 5. Estimated marginal posterior densities of additive effect for the relevant SNP on individual UFA

\begin{tabular}{|c|c|c|c|c|c|c|c|}
\hline Trait & Gene $^{1}$ & rs no. ${ }^{2}$ & Allele & Effect $^{3}$ & $\mathrm{HPD} 95^{4}$ & $\mathrm{PPN}^{5}$ & $\mathrm{~V}_{\mathrm{a}},{ }^{6} \%$ \\
\hline \multicolumn{8}{|l|}{ MUFA } \\
\hline \multirow[t]{2}{*}{ C10:1 cis-9 } & $C C L 3$ & rs109686238 & T vs. C & 0.009 & $0.001 ; 0.017$ & 0.992 & 3.8 \\
\hline & $A C A C A$ & rs110562092 & A vs. $G$ & -0.009 & $-0.015 ;-0.002$ & 0.994 & 2.4 \\
\hline $\mathrm{C} 14: 1$ cis-9 & $L P I N 1$ & rs136905033 & T vs. C & 0.078 & $0.001 ; 0.157$ & 0.974 & 3.5 \\
\hline \multirow[t]{2}{*}{$\mathrm{C} 16: 1$ cis-9 } & $P I$ & rs41257077 & A vs. $G$ & -0.041 & $-0.078 ;-0.003$ & 0.983 & 2.5 \\
\hline & $L P I N 1$ & rs136905033 & T vs. C & 0.094 & $0.010 ; 0.181$ & 0.984 & 6.6 \\
\hline C16:1 trans-9 & $\mathrm{FABP}_{4}$ & rs110757796 & A vs. $G$ & 0.006 & $0.001 ; 0.011$ & 0.989 & 1.0 \\
\hline \multirow[t]{4}{*}{$\mathrm{C} 17: 1$ cis-9 } & CCL3 & rs109686238 & T vs. C & -0.013 & $-0.020 ;-0.006$ & 1.000 & 0.3 \\
\hline & $A C A C A$ & rs110562092 & A vs. G & 0.006 & $0.001 ; 0.012$ & 0.977 & 0.1 \\
\hline & $L E P$ & rs110559656 & A vs. $G$ & 0.009 & $0.002 ; 0.017$ & 0.994 & 0.1 \\
\hline & $A D R B 2$ & rs132839139 & A vs. G & -0.042 & $-0.075 ;-0.010$ & 0.994 & 0.7 \\
\hline \multirow[t]{3}{*}{$\mathrm{C} 18: 1$ trans -4} & $C C L 2$ & rs41255713 & T vs. C & 0.003 & $0.001 ; 0.006$ & 0.986 & 0.3 \\
\hline & $F A B P 4$ & rs110757796 & A vs. $G$ & 0.004 & $0.001 ; 0.007$ & 0.988 & 0.4 \\
\hline & STAT5A & rs109578101 & T vs. C & -0.005 & $-0.010 ;-0.001$ & 0.984 & 0.5 \\
\hline \multirow[t]{2}{*}{ C18:1 trans $-6-8$} & $S T A T 1$ & rs43705173 & T vs. C & -0.006 & $-0.012 ;-0.001$ & 0.988 & 1.7 \\
\hline & $F G F 2$ & rs110937773 & A vs. $G$ & 0.005 & $0.001 ; 0.009$ & 0.975 & 1.2 \\
\hline C18:1 trans-9 & AGPAT6 & rs110445169 & T vs. C & -0.005 & $-0.010 ;-0.001$ & 0.980 & 1.2 \\
\hline \multirow[t]{2}{*}{$\mathrm{C} 18: 1$ trans -10} & CSN2 & rs43703011 & A vs. C & 0.014 & $0.001 ; 0.027$ & 0.984 & 6.9 \\
\hline & $G H R$ & rs109136815 & T vs. C & 0.011 & $0.002 ; 0.019$ & 0.993 & 5.1 \\
\hline C18:1 cis-9 & $L E P$ & rs110559656 & A vs. $G$ & 0.414 & $0.091 ; 0.739$ & 0.995 & 9.1 \\
\hline \multirow[t]{3}{*}{$\mathrm{C} 18: 1$ cis-12 } & STAT1 & rs 43705173 & T vs. C & -0.009 & $-0.016 ;-0.002$ & 0.995 & 3.8 \\
\hline & STAT1 & rs43706906 & $\mathrm{C}$ vs. $\mathrm{G}$ & -0.007 & $-0.013 ;-0.001$ & 0.981 & 2.4 \\
\hline & $A B C G 2$ & rs41577868 & T vs. G & -0.006 & $-0.013 ;-0.001$ & 0.978 & 1.8 \\
\hline \multirow[t]{3}{*}{ C18:1 trans-16 } & STAT1 & rs43705173 & T vs. C & -0.008 & $-0.014 ;-0.001$ & 0.990 & 3.0 \\
\hline & STAT1 & rs43706906 & $\mathrm{C}$ vs. $\mathrm{G}$ & -0.006 & $-0.012 ;-0.001$ & 0.981 & 1.8 \\
\hline & $C A R D 15$ & rs43710288 & T vs. A & -0.006 & $-0.013 ;-0.001$ & 0.979 & 1.7 \\
\hline \multirow[t]{2}{*}{$\mathrm{C} 20: 1$ cis -9} & GHR & rs109136815 & T vs. C & -0.004 & $-0.008 ;-0.001$ & 0.989 & 0.7 \\
\hline & $S C D 1$ & rs41255693 & T vs. C & 0.009 & $0.001 ; 0.018$ & 0.982 & 2.1 \\
\hline \multicolumn{8}{|l|}{ PUFA } \\
\hline \multirow[t]{3}{*}{ C18:2 cis-9,trans-11 (rumenic acid) } & $L E P$ & rs29004170 & $\mathrm{C}$ vs. $\mathrm{G}$ & -0.020 & $-0.033 ;-0.006$ & 0.998 & 6.5 \\
\hline & CSN2 & rs43703013 & G vs. C & -0.032 & $-0.056 ;-0.009$ & 0.996 & 9.2 \\
\hline & $P R L$ & rs110684599 & A vs. C & 0.036 & $0.014 ; 0.057$ & 0.999 & 16.2 \\
\hline $\mathrm{C} 18: 2$ trans-11,cis-15 & $L E P$ & rs29004170 & C vs. $\mathrm{G}$ & -0.008 & $-0.016 ;-0.001$ & 0.976 & 3.1 \\
\hline C18:2 trans -9, trans -12 & $P R L$ & rs211032652 & T vs. C & 0.014 & $0.009 ; 0.101$ & 0.988 & 1.5 \\
\hline \multirow[t]{2}{*}{$\mathrm{C} 18: 2$ cis -9, cis -12} & $F A B P 4$ & rs110757796 & A vs. G & 0.074 & $0.003 ; 0.145$ & 0.980 & 6.7 \\
\hline & LPIN1 & rs137457402 & T vs. G & -0.043 & $-0.077 ;-0.007$ & 0.992 & 4.1 \\
\hline $\mathrm{C} 18: 3$ cis-9,cis-12,cis-15 & LPIN1 & rs136905033 & T vs. C & -0.037 & $-0.072 ;-0.003$ & 0.982 & 8.2 \\
\hline \multirow[t]{2}{*}{$\mathrm{C} 18: 3$ cis- 9, trans -11, cis- 15} & PLCE1 & rs41624917 & T vs. C & 0.003 & $0.001 ; 0.006$ & 0.997 & 0.0 \\
\hline & STAT5A & rs137182814 & C vs. G & -0.043 & $-0.078 ;-0.010$ & 0.993 & 4.2 \\
\hline \multirow[t]{3}{*}{$\mathrm{C} 20: 3$ cis-8, cis-11, cis-14 } & STAT5A & rs137182814 & C vs. $\mathrm{G}$ & -0.047 & $-0.094 ;-0.001$ & 0.975 & 14.5 \\
\hline & STAT1 & rs43705173 & T vs. C & -0.005 & $-0.009 ;-0.001$ & 0.990 & 1.2 \\
\hline & STAT1 & rs43706906 & C vs. $\mathrm{G}$ & -0.005 & $-0.009 ;-0.001$ & 0.990 & 1.2 \\
\hline \multirow[t]{2}{*}{$\mathrm{C} 20: 4$ cis-5,cis-8,cis-11,cis-14 } & STAT1 & rs 43705173 & T vs. C & -0.005 & $-0.009 ;-0.001$ & 0.990 & 1.1 \\
\hline & STAT1 & rs43706906 & $\mathrm{C}$ vs. $\mathrm{G}$ & -0.005 & $-0.009 ;-0.001$ & 0.990 & 1.2 \\
\hline \multirow[t]{2}{*}{$\mathrm{C} 20: 5$ cis-5,cis-8,cis-11,cis-14,cis-17 } & $L E P R$ & rs43349286 & T vs. C & -0.003 & $-0.006 ;-0.001$ & 0.981 & 0.3 \\
\hline & $A C A C A$ & rs110562092 & A vs. G & -0.002 & $-0.004 ;-0.001$ & 0.988 & 0.2 \\
\hline \multirow[t]{2}{*}{$\mathrm{C} 22: 4$ cis- 7, cis- 10, cis- 13, cis- 16} & PLCE1 & rs41624917 & T vs. C & -0.002 & $-0.004 ;-0.001$ & 0.985 & 0.2 \\
\hline & $P I$ & rs41257077 & A vs. $G$ & 0.002 & $0.000 ; 0.004$ & 0.980 & 0.1 \\
\hline \multirow[t]{3}{*}{$\mathrm{C} 22: 5$ cis-7,cis-10,cis-13,cis-16,cis-19 } & $F G F 2$ & rs110937773 & A vs. $G$ & -0.003 & $-0.006 ;-0.001$ & 0.998 & 0.4 \\
\hline & $D G K G$ & rs41608610 & T vs. C & -0.006 & $-0.011 ;-0.002$ & 0.997 & 1.0 \\
\hline & STAT5A & rs109578101 & T vs. C & -0.008 & $-0.015 ;-0.001$ & 0.982 & 1.3 \\
\hline
\end{tabular}

${ }^{1}$ All gene symbols were defined in Cecchinato et al. (2014).

${ }^{2}$ http://www.ncbi.nlm.nih.gov/snp.

${ }^{3} \mathrm{SNP}$ were considered to have a relevant effect on the trait when the posterior mean of the additive effect did not include zero in the HPD interval.

${ }^{4}$ HPD95 = lower and upper bound of the $95 \%$ highest posterior density region.

${ }^{5} \mathrm{PPNO}=$ the probability of the additive effect to be over or below zero.

${ }^{6} \mathrm{~V}_{\mathrm{a}}=$ proportion (\%) of genetic variance explained by each SNP. 
Table 6. Estimated marginal posterior densities of additive and dominance effects for the relevant SNP on groups of fatty acids and unsaturation indices.

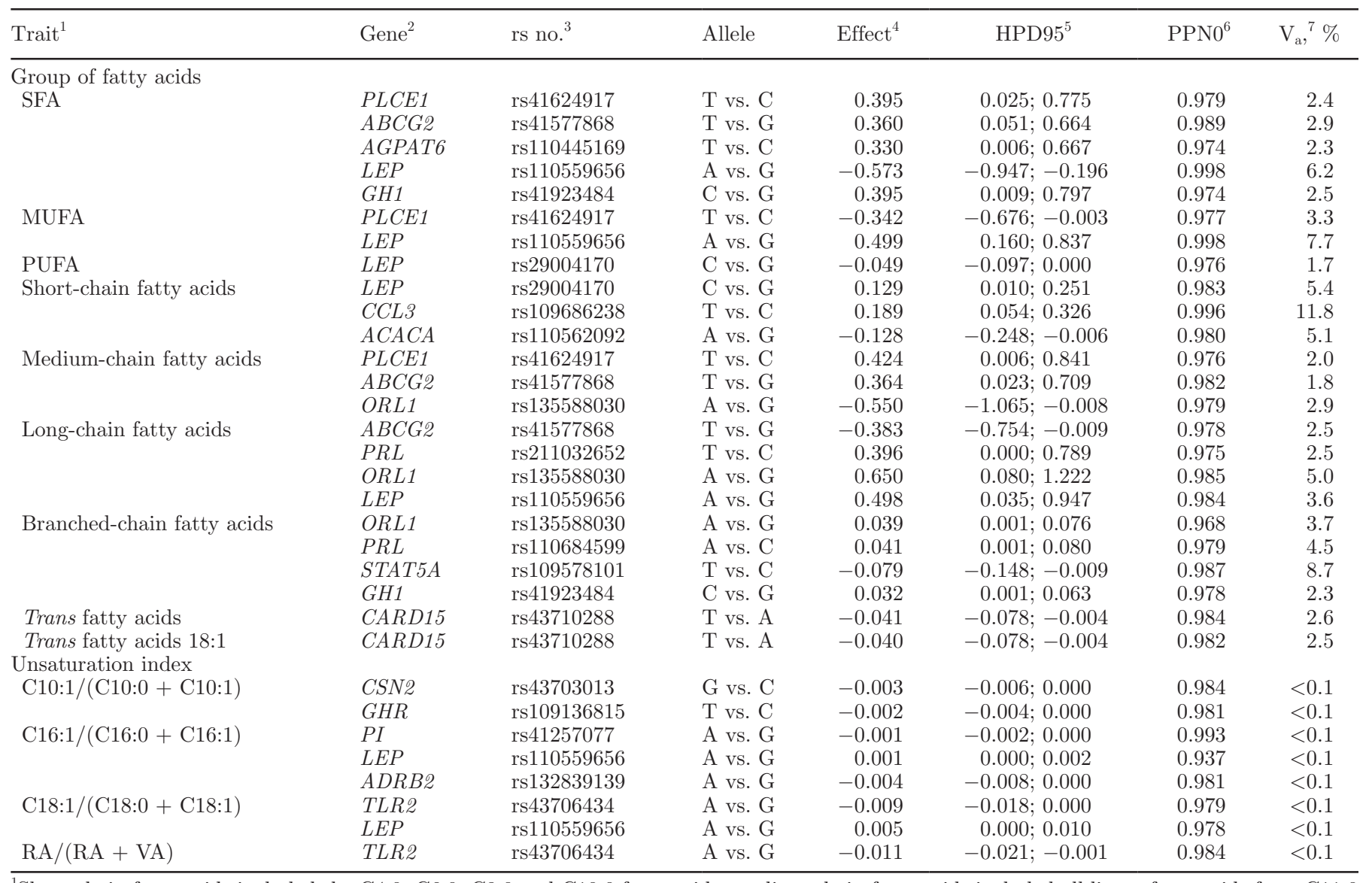

${ }^{1}$ Short-chain fatty acids included the C4:0, C6:0, C8:0 and C10:0 fatty acids; medium-chain fatty acids included all linear fatty acids from C11:0 to C16:1; long-chain fatty acids included all linear fatty acids from C17:0 to C24:0; trans fatty acids included all trans fatty acids; trans fatty acids C18:1 included all trans isomers of C18:1. RA = rumenic acid; VA = vaccenic acid

${ }^{2}$ All gene symbols were defined in Cecchinato et al. (2014).

${ }^{3}$ http://www.ncbi.nlm.nih.gov/snp.

${ }^{4} \mathrm{SNP}$ were considered to have a relevant effect on the trait when the posterior mean of the additive effect did not include zero in the HPD interval.

${ }^{5}$ HPD95 = lower and upper bound of the $95 \%$ highest posterior density region.

${ }^{6} \mathrm{PPN} 0=$ the probability of the additive effect to be over or below zero.

${ }^{7} \mathrm{~V}_{\mathrm{a}}=$ proportion (\%) of genetic variance explained by each SNP.

a SNP in the LEP gene (g.1180C $>\mathrm{T}$ ) and the analyzed fatty acid traits in 3 different bovine breeds.

\section{Prolactin}

As expected, the PRL gene on BTA23 showed relevant associations with some of the milk fatty acid traits considered (7 traits; Table 7 ). Prolactin plays a critical role in mammary gland development, lactogenesis, and galactopoiesis. The mechanism responsible for the effects of PRL on milk fatty acid composition is not completely known, but it seems to involve signal transducer and activator of transcription (STAT)5A, which is regulated by PRL (Welte et al., 1994). However, an investigation of the PRL-mediated regulation of lipid biosynthesis genes in the mouse demonstrated that the expression of several transcription factors (including SREBP1) and lipogenic genes involved in de novo fatty acids synthesis, triglyceride synthesis, and $\beta$-oxidation were rapidly and coordinately decreased in lactating mammary gland cells via suppression of serum PRL. Moreover, PRL seems to be involved in cytosolic lipid droplet formation (Rudolph et al., 2011).

Here, we found relevant associations of $2 \mathrm{SNP}$ of the PRL gene (rs110684599 and rs211032652) with the contents of 7 fatty acids. The $A$ allele of rs110684599 was 
positively associated with $\mathrm{RA}\left(\mathrm{V}_{\mathrm{a}}=16.2 \%\right)$, C14:0 iso $\left(\mathrm{V}_{\mathrm{a}}=3.8 \%\right)$, and $\mathrm{C} 15: 0$ iso $\left(\mathrm{V}_{\mathrm{a}}=8.4 \%\right)$, whereas the $T$ allele of rs211032652 was positively associated with C18:2 trans-9,trans-12 $\left(\mathrm{V}_{\mathrm{a}}=1.5 \%\right)$, long-chain SFA $\left[\mathrm{C} 18: 0\left(\mathrm{~V}_{\mathrm{a}}=2.8 \%\right)\right.$ and $\left.\mathrm{C} 20: 0\left(\mathrm{~V}_{\mathrm{a}}=1.4 \%\right)\right]$, and $\mathrm{C} 17: 0$ anteiso $\left(\mathrm{V}_{\mathrm{a}}=6.7 \%\right)$. To our knowledge, this is the first time that a SNP of the $P R L$ gene has been associated with RA, for which it explains more than $15 \%$ of genetic variance of the trait. We previously reported that another SNP of the PRL gene (rs109428015) was relevantly associated with some milk technological traits, such as the time required for curd firming and time to maximum curd firmness (Cecchinato et al., 2015). A previous study found a different $P R L$ mutation, g.8398G $>$ A, showing an effect (albeit not significant) on C10:0 and C12:1 in Dutch Holstein-Friesian cows (Schennink et al., 2009). On the other hand, studies on genome-wide associations with milk fatty acid traits in different populations of dairy cattle did not detect any QTL on BTA23 close to the PRL gene (Bouwman et al., 2011; Buitenhuis et al., 2014; Li et al., 2014). These contrasting results could be explained by a low frequency of SNP in PRL in Holsteins or by the presence of allele-specific populations.

It is worth mentioning that Nafikov and colleagues (2013a) found significant associations of haplotypes in the sterol regulatory element binding transcription factor 1 (SREBF1) with milk production and variations in lauric acid (12:0) and myristic acid (14:0) concentrations in milk. In particular, a tag SNP (SREBF1:g.13495T >C) caused the Pro852Leu mutation in the regulatory C-terminal domain of SREBP1, and Pro852 was found to be highly conserved among multiple mammalian species including cow. Despite this, the authors provided evidence for the prevalence of Leu852 in their population as a probable result of an- imal selection. Due to the putative functional relationships between PRL and SREBP1, we cannot exclude the possibility that the observed effects on milk fatty acids composition might be due to SNP in SREBF1 rather than in $P R L$.

\section{STAT5A}

Signal transducer and activator of transcription 5A (STAT5A), which is located on BTA19, encodes a transcription factor that plays an important role in lactogenesis, as PRL imparts its effects on milk protein and lipid synthesis mainly through the Jak2/STAT5 signaling pathway (Rudolph et al., 2011). We found that 2 polymorphisms (rs109578101 and rs137182814) in STAT5A relevantly influenced 7 fatty acids, including some branched-chain fatty acids (BCFA). The $T$ allele of rs109578101 negatively influenced C17:0 anteiso $\left(\mathrm{V}_{\mathrm{a}}=18.8 \%\right)$, whereas the $C$ allele of rs137182814 showed a positive association with $\mathrm{C} 17: 0$ iso $\left(\mathrm{V}_{\mathrm{a}}=\right.$ $12.8 \%$ ) and negative associations with 2 PUFA: C18:3 cis-9,trans-11,cis-15 and C20:3 cis-8,cis-11,cis-14 ( $\mathrm{V}_{\text {a }}$ $=4.2$ and $14.5 \%$, respectively). A negative association was also found between the $T$ allele of rs109578101 and $\mathrm{C} 22: 5$ cis-7,cis-10,cis-13,cis-16,cis-19 (Table 5). We previously reported a relevant association between STAT5A rs109578101 and asymptotic potential curd firmness (Cecchinato et al., 2015). In Dutch HolsteinFriesian cows, a STAT5A polymorphism (g.9501G $>$ A) was found to be associated with medium-chain fatty acids (MCFA) and desaturase indices, with the $A$ allele showing positive associations with $\mathrm{C} 10: 0$ and $\mathrm{C} 14: 0$, and negative associations with the monounsaturated MCFA and desaturase indices (Schennink et al., 2009). Interestingly, a major QTL for milk fat content and fatty acids in bovine milk was previously detected on

Table 7. List of the most representative candidate genes

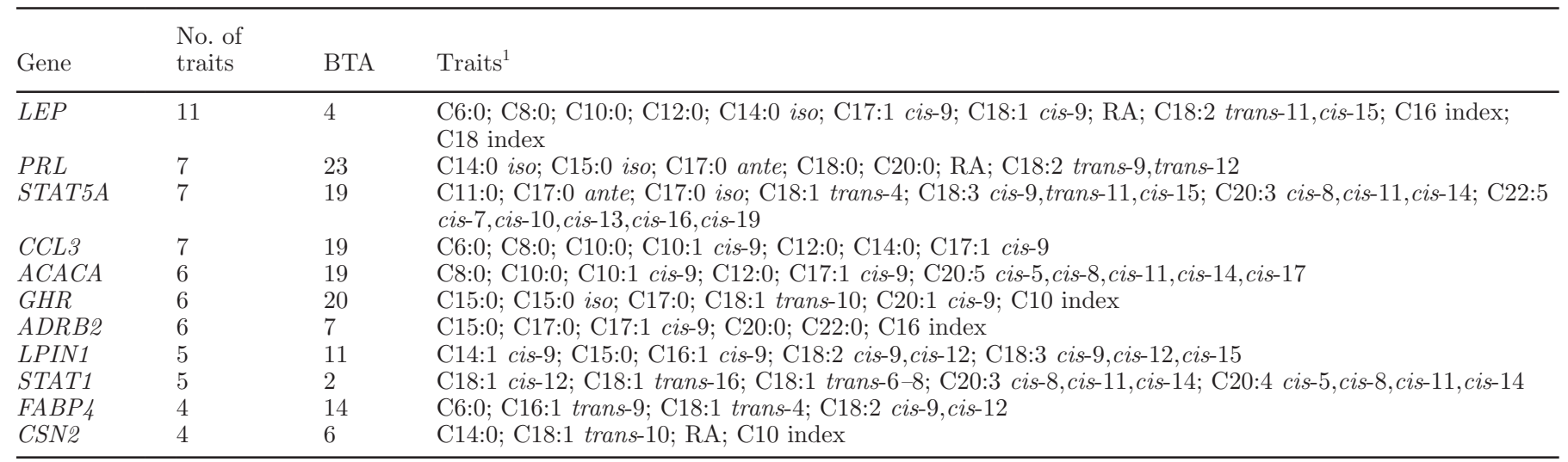

${ }^{1} \mathrm{RA}=$ rumenic acid. 
BTA19 in Holstein cows (Bouwman et al., 2011, 2012). In particular, significant associations were found for C4:0, C8:0 C10:0, C12:0, and C14:0 and SNP located in the region between 52.1 and $55.1 \mathrm{Mb}$ on BTA19. Stoop et al. (2009) found a putative QTL close to STAT5A (which is located at around $43 \mathrm{Mb}$ ) that was positively associated with C14:0 content in a Dutch Holstein population. In the Brown Swiss breed, major chromosomal regions on BTA19 showed significant associations with RA, including significant SNP close to STAT5A (Strillacci et al., 2014).

\section{Chemokine C-C Motif Ligand 3}

The chemokine $\mathrm{C}-\mathrm{C}$ motif ligand $(C C L) 3$ gene on BTA19 encodes a protein that contributes to inflammatory responses by binding the receptors, chemokine (C-C motif) receptor (CCR)1, CCR4, and CCR5. Recently, CCL3 was also associated with obesity in humans (Muñoz and Costa, 2013). Here, we found a single SNP on CCL3 (rs109686238) that was relevantly associated with 7 fatty acid traits. The $T$ allele showed a positive association with the SCFA concentration $\left(\mathrm{V}_{\mathrm{a}}\right.$ $=11.8 \%)$, most prominently with $\mathrm{C} 10: 0\left(\mathrm{~V}_{\mathrm{a}}=5.8 \%\right)$. A positive association was also observed with $\mathrm{C} 12: 0$ and C14:0 $\left(\mathrm{V}_{\mathrm{a}}=4.6\right.$ and $5.2 \%$, respectively). The $T$ allele of rs109686238 of CCL3 generally increased the content of de novo fatty acids from C6:0 to C14:0. Meanwhile, the $T$ allele of this SNP was negatively associated with 2 products of the SCD enzyme: C10:1 cis-9 and C17:1 cis-9. We are unaware of any previous report examining $C C L 3$ gene polymorphisms and the effects on the inflammatory response, so we are unable to evaluate whether the positive association of the $T$ allele with de novo fatty acids could also be associated with the main function of the gene.

\section{Acetyl-CoA Carboxylase $\alpha$}

The acetyl-CoA carboxylase $\alpha(A C A C A)$ gene on BTA19 encodes an enzyme that catalyzes the carboxylation of acetyl-CoA to malonyl-CoA, leading to the biosynthesis of de novo fatty acids from C4:0 to C16:0 in the mammary gland (Chilliard et al., 2000). Here, we report that rs110562092 of $A C A C A$ is associated with some de novo fatty acids. In particular, a negative association was found between the $A$ allele and the concentrations of $\mathrm{C} 8: 0, \mathrm{C} 10: 0$, and $\mathrm{C} 12: 0$ in milk fat $\left(\mathrm{V}_{\mathrm{a}}=5.2,2.5\right.$, and $2.3 \%$, respectively). A slight but relevant negative effect on the content of n-3 EPA (C20:5 cis-5,cis-8,cis-11,cis-14,cis-17) was also detected $\left(\mathrm{V}_{\mathrm{a}}=0.2 \%\right)$. Interestingly, polymorphisms in CCL3 and $A C A C A$ were found to affect largely the same fatty acids, and the 2 genes are located relatively close together on BTA19 (around 14.6 and $13.8 \mathrm{Mb}$, respectively). However, analyses performed with Haploview software (Barrett et al., 2005) to check for the existence of linkage disequilibrium between the 2 SNP in these genes showed that they were completely independent in the sample population (data not shown). Matsumoto and colleagues (2012) showed that $3 \mathrm{SNP}$ on $A C A C A$ influenced fatty acid composition in the milk of Holstein cows (in particular, the combined profile of C14:0, C16:0, and C18:0). Similarly, Li et al. (2014) found that a QTL in $A C A C A$ was significantly associated with C10:0 content in a Chinese Holstein population. Strillacci and colleagues (2014) found significant associations between SNP close to $A C A C A(13.7 \mathrm{Mb})$ and phenotypic variations in RA.

\section{Growth Hormone Receptor}

Growth hormone receptor $(G H R)$ is located on BTA20 and encodes a member of the type I cytokine receptor family of growth hormone receptors. It is considered a strong positional and functional candidate gene for association with milk fatty acid traits because it is located very close to a QTL previously shown to affect milk production traits (Blott et al., 2003). Indeed, we found that the $T$ allele of GHR rs109136815 was positively associated with the concentration of 5 fatty acids in milk fat. The most relevant effects were observed for C15:0 $\left(\mathrm{V}_{\mathrm{a}}=3.1 \%\right), \mathrm{C} 17: 0\left(\mathrm{~V}_{\mathrm{a}}=4.2 \%\right)$, and $\mathrm{C} 18: 1$ trans $-10\left(\mathrm{~V}_{\mathrm{a}}=5.1 \%\right)$. The odd-chain fatty acids (such as C15:0 and C17:0) are produced by elongation of propionate or valerate by rumen microbes (Fievez et al., 2012), so the relationship with this $G H R$ polymorphism is likely to be indirect. Rumen microbes are also responsible for the synthesis of C18:1 trans-10, which is an intermediate of the biohydrogenation of dietary PUFA. The role of C18:1 trans-10 in milk fat synthesis is not yet completely understood, but this fatty acid is likely to be involved (along with other rumen biohydrogenation intermediates) in modulating the expression of several genes involved in mammary lipid metabolism (Shingfield et al., 2013). To our knowledge, the literature does not contain any information regarding a relationship between C18:1 trans-10 and GHR. However, $\mathrm{Li}$ and colleagues (2014) found a significant association between one SNP located within GHR and the C18:0 content of milk from Chinese Holsteins.

Finally, the GHR polymorphism had only a trivial effect on the C10 index $\left(\mathrm{V}_{\mathrm{a}}<0.1 \%\right.$; Tables 6 and 7$)$.

\section{Adrenoceptor $\beta 2$}

Adrenoceptor $\beta 2$ (ADRB2), which is located on BTA7, encodes the $\beta 2$ adrenergic receptor. This recep- 
tor is part of the adrenergic system, which stimulates lipid mobilization in adipose tissue (Kurokawa et al., 2008). The transcription of $A D B R 2$ has been shown to increase during the transition phase of dairy cows, as lipolysis is strongly related to the activation of ADRB2 via norepinephrine produced by the sympathetic nervous system (Sumner-Thomson et al., 2011).

In the present work, we observed that the $A$ allele of $A D R B 2$ rs132839139 was negatively associated with the concentrations of some long-chain SFA in milk fat, such as $\mathrm{C} 17: 0\left(\mathrm{~V}_{\mathrm{a}}=16.0 \%\right), \mathrm{C} 20: 0\left(\mathrm{~V}_{\mathrm{a}}=4.6 \%\right)$, and $\mathrm{C} 22: 0\left(\mathrm{~V}_{\mathrm{a}}=1.9 \%\right)$. A relevant negative association was previously found between the same allele and MUN in Brown Swiss cows (Cecchinato et al., 2014). No association was found with respect to the main fatty acids involved in lipid mobilization, such as C16:0, C18:0, and C18:1. However, the ADRB2 polymorphism showed a relevant negative association with the $\mathrm{C} 16$ index $\left(\mathrm{V}_{\mathrm{a}}=\right.$ $0.001 \%)$.

\section{Lipin 1}

The lipin 1 (LPIN1) gene on BTA11 encodes the enzyme that cleaves the $\mathrm{P}$ group of phosphatidic acid during its conversion to diacylglycerol. The LPIN1 gene is the major isoform expressed in the bovine mammary gland, and its expression increased more than 3-fold during the first phase of lactation (Bionaz and Loor, 2008; Nafikov et al., 2014). It is known to be directly involved in milk fat synthesis and in regulating the transcription of other genes involved in milk fat synthesis (Bionaz and Loor, 2008). In the present work, we found that 2 SNP of LPIN1 were associated with 5 fatty acids: C14:1 cis-9, C15:0, C16:1 cis-9, C18:2 cis-9,cis-12, and C18:3 cis-9,cis-12,cis-15 (Table 6). The $T$ allele of rs136905033 was positively associated with C14:1 cis$9\left(\mathrm{~V}_{\mathrm{a}}=3.5 \%\right), \mathrm{C} 15: 0\left(\mathrm{~V}_{\mathrm{a}}=12.3 \%\right)$, and $\mathrm{C} 16: 1$ cis-9 $\left(\mathrm{V}_{\mathrm{a}}=6.6 \%\right)$, whereas it was negatively associated with $\alpha$-linolenic acid (C18:3 cis-9,cis-12,cis- $15 ; \mathrm{V}_{\mathrm{a}}=8.2 \%$ ). The $T$ allele of the other SNP (rs137457402), which has a relatively high MAF (0.43; Cecchinato et al., 2014), was negatively associated with the concentration of linoleic acid in milk fat (C18:2 cis-9,cis-12; $\mathrm{V}_{\text {a }}$ $=4.1 \%$ ). These alleles may thus negatively affect milk fatty acid composition through their associations with lower amounts of PUFA in milk fat. On the other hand, the positive associations of the $T$ allele of rs 136905033 with C14:1 cis-9 and C16:1 cis-9 suggest that this SNP may play a positive role in the biosynthetic activity of the mammary gland, where these 2 fatty acids are mainly produced (Mele et al., 2007). Results obtained in studies on human and animal models identified and characterized C16:1 cis-9 as a novel lipokine or lipid hormone (German, 2011).
Nafikov et al. (2014) did not observe any significant association between LPIN1 haplotypes and the milk fatty acid composition in Holstein cows.

\section{STAT1}

The STAT1 gene, which is located on BTA2, is constitutively expressed in the mammary gland at constant levels through pregnancy, lactation, and involution. The STAT1 protein is involved in the development and differentiation of the mammary gland, and it was shown to play a particularly important role in regulating the transcription of genes involved in milk protein synthesis and fat metabolism in Holstein cows (Cobanoglu et al., 2006).

We found that 2 SNP (rs43705173 and rs43706906) in the STAT1 gene were associated with the concentration of 5 fatty acids. The $T$ allele of rs 43705173 and the $C$ allele of rs43706906 were associated with slight reductions of $\mathrm{C} 18: 1$ trans- $16\left(\mathrm{~V}_{\mathrm{a}}=3.0\right.$ and $1.8 \%$, respectively), C18:1 cis-12 ( $\mathrm{V}_{\mathrm{a}}=3.8$ and $2.4 \%$, respectively), C20:3 cis-8, cis-11,cis-14 ( $\mathrm{V}_{\mathrm{a}}=1.2 \%$ for both SNP), and $\mathrm{C} 20: 4$ cis-5, cis-8, cis-11,cis-14 $\left(\mathrm{V}_{\mathrm{a}}=1.1\right.$ and $1.2 \%$, respectively). Given these effects on the same fatty acid traits, the existence of linkage disequilibrium between these SNP on STAT1 gene was evaluated and confirmed by Haploview analyses ( $\mathrm{D}^{\prime}$, the normalized linkage disequilibrium coefficient, $=0.98$; data not shown). In the case of C18:1 trans-6-8, only rs43705173 had a relevant effect, showing a negative association with the $T$ allele. The effect on trans fatty acids was probably only indirect, as this class of fatty acids originates in the rumen during the biohydrogenation process. In the cases of $\mathrm{C} 20: 3$ cis-8,cis-11,cis-14 and $\mathrm{C} 20: 4$ cis-5, cis8,cis-11,cis-14, the $2 \mathrm{SNP}$ for STAT1 and the $1 \mathrm{SNP}$ for STAT5A were the only SNP that had relevant effects. This suggests that elongation of n-3 and n-6 fatty acids is probably related to these $2 S T A T$ genes. The literature includes few studies on the effects of STAT1 polymorphisms on milk fatty acid composition. Two SNP located $0.50 \mathrm{Mb}$ away from the STAT1 gene were previously associated with the C18:0 and C18 index in Chinese Holstein (Li et al., 2014).

\section{Fatty Acid Binding Protein 4}

The fatty acid binding protein $(F A B P) 4$ gene, which is located on BTA14, encodes an intracellular protein that binds nonesterified saturated and unsaturated fatty acids, eicosanoids, and other lipids to transport or store them inside a cell (Bionaz and Loor, 2008). Several papers have demonstrated significant associations between genetic polymorphisms in FABP 4 and the fatty acid composition in adipocytes (Michal et al., 
2006; Pannier et al., 2010). The FABP4 gene is expressed in the mammary gland, along with $F A B P 3$ and $F A B P 5$, but little information is available regarding the function of FABP4 in the mammary gland.

Our results showed that the $A$ allele of FABP4 rs110757796 induced a slight increase in the amount of linoleic acid (C18:2 cis-9, cis-12; $\left.\mathrm{V}_{\mathrm{a}}=6.7 \%\right)$. Interestingly, a previous study showed that the MAF was relatively low for this allele $(0.16$; Cecchinato et al., 2014), suggesting that it may be possible to develop breeding strategies to increase its frequency. A positive association was also observed with $\mathrm{C} 6: 0\left(\mathrm{~V}_{\mathrm{a}}=14.1 \%\right)$, and near-trivial positive associations were found for $\mathrm{C} 16: 1$ trans -9 and $\mathrm{C} 18: 1$ trans $-4\left(\mathrm{~V}_{\mathrm{a}}=1.0\right.$ and $0.4 \%$, respectively). Marchitelli et al. (2013) found that FABP4 was the most important gene affecting milk fatty acid composition in different bovine breeds. In particular, FABP 4 g.2989A $>$ G showed negative effects on MCFA and positive effects on long-chain fatty acids (LCFA). A FABP 4 haplotype was found to be negatively associated with SFA, SFA:UFA ratio, C12:0, and C14:0, whereas it was positively associated with UFA and MUFA (Nafikov et al., 2013b).

\section{$\beta$-Casein}

The CSN2 gene on BTA6 is highly polymorphic (Chessa et al., 2013) and encodes $\beta-\mathrm{CN}$, one of the main caseins present in milk proteins. In the present study, we found that $2 \mathrm{SNP}$ in CSN2 had relevant effects on milk fatty acid composition: rs43703013 and rs43703011. In the first case, a negative association was revealed between the $G$ allele and the concentration of $\mathrm{RA}$ in milk fat $\left(\mathrm{V}_{\mathrm{a}}=9.2 \%\right)$. Bouwman et al. (2011) previously reported that a region of BTA6 between 111.2 and $115.6 \mathrm{Mb}$ harbored SNP with a relevant effect on the concentration of RA in milk fat. The $A$ allele of CSN2 rs43703011 showed a negative effect on the concentration of $\mathrm{C} 14: 0$ in milk fat $\left(\mathrm{V}_{\mathrm{a}}=7.8 \%\right)$, a positive effect on the concentration of C18:1 trans-10 in milk fat $\left(\mathrm{V}_{\mathrm{a}}=6.9 \%\right)$, and a relevant negative association with the $\mathrm{C} 10$ index $\left(\mathrm{V}_{\mathrm{a}}<0.1 \%\right)$.

Polymorphisms in CSN2 have been reported to have relevant effects on milk and protein yield. In dairy goats, CSN2 haplotypes were shown to affect the milk fat percentage (Dagnachew and Ådnøy, 2014). Moreover, a previous genome-wide association suggested that a region on BTA6 close to candidate genes [e.g., peroxisome proliferator-activated receptor gamma and coactivator $1 \alpha(P A R G C 1 A)]$ harbored SNP that have significant effects on C6:0, C8:0 (Stoop et al., 2009), C12:1 cis-9, C14:1 cis-9, and C16:1 cis-9 (Bouwman et al., 2011).

\section{Stearoyl-CoA Desaturase 1}

Although SCD1 was not included in the list of the most representative genes (in terms of number of relevant associations; Table 7), SCD1 is another gene having a pivotal role in fatty acid metabolism and is a well-known candidate gene for association analyses with fatty acids. The SCD1 gene on BTA26 encodes the SCD1 enzyme (also known as $\Delta^{9}$-desaturase), which introduces a double bond at the $\Delta^{9}$-position of a large spectrum of fatty acids, including acyl-CoA of C14:0, C16:0, C18:0, and C18:1 trans-11, which are converted into $\mathrm{C} 14: 1$ cis-9, C16:1 cis-9, C18:1 cis-9, and RA, respectively (Mele et al., 2007). In the present study, the $T$ allele of $S C D 1$ rs 41255693 , which causes an A-to-V amino acid substitution (SCD1 A293V) at $21.1 \mathrm{Mb}$, was positively associated with the concentrations of both $\mathrm{C} 22: 0$ and $\mathrm{C} 20: 1$ cis-9 in milk fat $\left(\mathrm{V}_{\mathrm{a}}=\right.$ 1.3 and $2.1 \%$, respectively). In a previous study from our group, the same SNP of the $S C D 1$ gene was also relevantly associated with an increase in fat percentage (Cecchinato et al., 2014). Moreover, several studies from other groups have reported polymorphisms in both exonic and intronic regions of the gene in Holsteins, and showed that they had some relevant effects on the desaturation index and the milk contents of RA and several SFA (Mele et al., 2007; Schennink et al., 2008). Stearoyl-CoA desaturase plays a major role in determining the milk contents of MUFA, primarily oleic acid, as well as the CLA content of milk fat. Surprisingly, we did not find any relevant association for additive genetic effect and the main products of SCD enzyme activity reported above. In a different Brown Swiss population, Conte et al. (2010) found that the same SNP had significant effects on C14 index and the milk content of C14:1 cis-9. However, that study was performed on a much smaller number of animals (351 vs. 1,158 in the present work) and using a different statistical approach. Besides, when specifically looking at the content of C14:1 cis-9 and the estimated C14 index, we observed similar values between the homozygous $A A$ and the heterozygous $A V$, which both differed from the homozygous $V V$, suggesting the absence of an allele substitution effect. Interestingly, we observed relevant dominance effects (d) for rs136334180 on C18 and CLA indices $(\mathrm{d}=0.01), \mathrm{C} 18: 0(\mathrm{~d}=-0.23)$, RA $(\mathrm{d}=0.02)$, PUFA $(\mathrm{d}=0.08), \mathrm{C} 20: 1$ cis-9 $(\mathrm{d}<0.01)$ and for rs41255693 on C16:0 iso $(\mathrm{d}=-0.02)$ and $\mathrm{C} 20: 1$ cis-9 $(\mathrm{d}=-0.01$; data not shown), supporting the hypothesis that the effects of the $A$ allele (rs136334180) and $T$ allele (rs41255693) are not additive in the Brown Swiss population. The balance between the 2 alleles of SNP rs41255693 differed between Holsteins and our 
Brown Swiss population, in which the SNP was nearly monomorphic (Conte et al., 2010; Cecchinato et al., 2014). Interestingly, the dominance effects of SNP in the $S C D$ gene were found to be relevant for milk yield, fat yield, protein yield, and protein percentage in Chinese Holstein (Alim et al., 2012).

\section{Other Genes}

The results of our association analyses for other tested candidate genes are briefly reported below. Considering fatty acid groups and indices, relevant associations were found as follows: for SFA with SNP related to the genes encoding ATP-binding cassette sub-family G member 2 (ABCG2), 1-acylglycerol-3-phosphate Oacyltransferase 6 (AGPAT6), growth hormone 1 (GH1), and phospholipase C epsilon 1 (PLCE1); for MUFA with a SNP of $P L C E 1$; for BCFA with polymorphisms of $G H 1$ and the gene encoding opiate receptor-like 1 (ORL1); for MCFA with polymorphisms of $A B C G 2$, ORL1 and PLCE1; for LCFA with SNP of $A B C G 2$, the gene encoding toll-like receptor 2 (TLR2), and ORL1; for trans fatty acids with a SNP of CARD15; for the C16 index with a SNP of the gene encoding serpin peptidase inhibitor $1(P I)$; and for the C18:0 and CLA indices with a polymorphism of TLR2.

Regarding individual fatty acids, relevant associations were found as follows: for C4:0 with polymorphisms of both the gene encoding liver $\mathrm{X}$ nuclear receptor $\alpha$ variant $1(L X R \alpha)$ and PPARGC1A; for C13:0 with SNP of the genes encoding CCL2 (CCL2) and CCR2 (CCR2); for C15:0 iso with a polymorphism of the gene encoding xanthine dehydrogenase $(X D H)$; for $\mathrm{C} 17: 0$ iso and $\mathrm{C} 22: 0$ with a SNP of the gene encoding $\alpha_{\mathrm{S} 1}-\mathrm{CN}$ (CSN1S1); for C18:1 trans-6-8 and C22:5 cis-7,cis10,cis-13,cis-16,cis-19 with a SNP of the gene encoding fibroblast growth factor 2 (FGF2); for C20:0 with a $\mathrm{SNP}$ of the gene encoding $\mathrm{k}-\mathrm{CN}$ (CSN3); for C22:5 cis7,cis-10,cis-13,cis-16,cis-19 with a polymorphism of the gene encoding diacylglycerol kinase gamma $(D G K G)$; and for C20:5 cis-5,cis-8,cis-11,cis-14,cis-17 with a SNP of the gene encoding leptin receptor $(L E P R)$.

For many of these genes, the associations of SNP with milk technological and production traits were previously reported in the same population of Brown Swiss cows, such as the SNP of CARD15 (MUN, \% casein, $\%$ protein), GH1 (rennet coagulation time), and CSN3 (\% lactose; Cecchinato et al., 2014, 2015). Several other associated SNP are located in genes previously identified as potential candidate genes in association analysis with milk fatty acids or in genes known to be involved in milk production or fat synthesis (e.g., $A B C G 2, G H 1$, PPARGC1A, AGPAT6 and FGF2; Bionaz and Loor, 2008; Wang et al., 2008; Bouwman et al., 2011).
In our analyses, we did not include some wellknown candidate genes for association analyses with milk fatty acids (i.e., DGAT1 or FASN). In particular, $D G A T 1$ was not considered in the analysis because it is nearly monomorphic in Italian Brown Swiss (Conte et al., 2010), whereas FASN was included in the initial panel of tested SNP but was not genotyped due to a designability rank equal to 0.5 (moderate success rate, Supplemental Data file; http://dx.doi.org/10.3168/ jds.2015-10420).

We found many relevant associations for LCFA, which are derived from circulating plasma lipids and originate from the diet, ruminal metabolic processes, and from endogenous lipids. However, when comparing the number of relevant SNP for fatty acids contents with $<16 \mathrm{C}$ and $>16 \mathrm{C}$, balanced percentages on the total of SNP were found (42 and $58 \%$, respectively; data not shown). It is worth noting that evidence of QTL for LCFA in bovine milk have been presented by Schennink et al. (2009), supporting the hypothesis that a genetic component exists that influences variation in these fatty acids by affecting their synthesis or metabolism (i.e., uptake, desaturation, esterification, biohydrogenation, and elongation). The existence of genetic variation for some LCFA was also evidenced by the high heritability values observed for C18:1 and CLA by Bouwman et al. (2014).

In this work, we used a targeted list of SNP in candidate genes, and sequence data are required to identify causal mutations responsible for the phenotype variations and thereby to potentially use these SNP in breeding programs. However, our results provide enough evidence to test these SNP in a genomic prediction approach based on the biological understanding of the phenotype compared with an "anonymous" approach without prior information.

\section{CONCLUSIONS}

In the present work, we used GC to perform association analyses between 51 previously selected SNP and 61 fatty acid traits in bovine milk samples. In terms of the fatty acid traits analyzed, this is the most comprehensive study performed to date on the milk fatty acid profile using a candidate gene approach. Most of the studied fatty acid traits $(\sim 81 \%)$ were relevantly associated with multiple SNP. The highest number of relevant associations was found mostly for SNP located in genes involved in fat synthesis or metabolism, linked to or contained in previously identified QTL for fat yield or content, or previously identified as candidate genes in association analyses with milk fatty acid profiles in other cow breeds. In particular, we found relevant associations with SNP located on chromosome 
BTA19. Two candidate genes located on BTA19 (CCL3 and $A C A C A$ ) were relevantly associated with de novo SCFA and MCFA, thus explaining the high heritability values previously found for these fatty acids (with the exception of C6:0). Two other genes on BTA19 (CCL2 and GH1) showed relevant association with SFA and BCFA. Among the candidate genes, LEP deserves particular attention, as it showed the greatest number of relevant associations (11). In addition, the 2 SNP of the $L E P$ gene were associated with reductions of SFA and increases of MUFA and PUFA (including RA); according to current findings, this is likely to enhance milk quality. Recent studies have provided new insights into the importance and biological activity of specific fatty acids. Thus, it is important that the present study include macro-categories and representative fatty acids, as well as individual fatty acids that are present in small amounts but which may have potential beneficial effects for human health. Our identification of SNP in genes responsible for variations in bovine milk fat composition therefore provides worthwhile information that could be used in breeding programs to tailor the fatty acid content of bovine milk.

\section{ACKNOWLEDGMENTS}

The present study is part of the Cowability-Cowplus project. The authors thank the Autonomous Province of Trento for funding the project; the Italian Brown Swiss Cattle Breeders Association (ANARB, Verona, Italy) for supplying pedigree information; the Superbrown Consortium of Bolzano and Trento (Trento, Italy) for supporting our sampling and recording activities; and Fabio Maretto, Department of Agronomy, Food, Natural Resources, Animals and Environment (DAFNAE), University of Padova (Padova, Italy) for cooperating in the initial data analyses. The authors also acknowledge M. L. Dettori (Dipartimento di Medicina Veterinaria, Università degli Studi di Sassari, Sassari, Italy) and J. Casellas (Departament de Ciencia Animal i dels Aliments, Universitat Autonoma de Barcelona, Bellaterra, Spain) for useful comments and suggestions.

\section{REFERENCES}

Alim, M. A., Y. P. Fan, X. P. Wu, Y. Xie, Y. Zhang, S. L. Zhang, D. X. Sun, Y. Zhang, Q. Zhang, L. Liu, and G. Guo. 2012. Genetic effects of stearoyl-coenzyme A desaturase (SCD) polymorphism on milk production traits in the Chinese dairy population. Mol. Biol. Rep. 39:8733-8740.

Barrett, J. C., B. Fry, J. Maller, and M. J. Daly. 2005. Haploview: Analysis and visualization of LD and haplotype maps. Bioinformatics 21:263-265.

Bastin, C., H. Soyeurt, and N. Gengler. 2013. Genetic parameters of milk production traits and fatty acid contents in milk for Holstein cows in parity 1-3. J. Anim. Breed. Genet. 130:118-127.
Bionaz, M., and J. J. Loor. 2008. ACSL1, AGPAT6, FABP3, LPIN1, and SLC27A6 are the most abundant isoforms in bovine mammary tissue and their expression is affected by stage of lactation. J. Nutr. 138:1019-1024.

Blott, S., J. J. Kim, S. Moisio, A. Schmidt-Kuntzel, A. Cornet, P. Berzi, N. Cambisano, C. Ford, B. Grisart, D. Johnson, L. Karim, P. Simon, R. Snell, R. Spelman, J. Wong, J. Vilkki, M. Georges, F. Farnir, and W. Coppieters. 2003. Molecular dissection of a quantitative trait locus: A phenylalanine-to-tyrosine substitution in the transmembrane domain of the bovine growth hormone receptor is associated with a major effect on milk yield and composition. Genetics 163:253-266.

Borreani, G., M. Coppa, A. Revello-Chion, L. Comino, D. Giaccone, A. Ferlay, and E. Tabacco. 2013. Effect of different feeding strategies in intensive dairy farming systems on milk fatty acid profiles, and implications on feeding costs in Italy. J. Dairy Sci. 96:6840-6855.

Bouwman, A. C., H. Bovenhuis, M. H. Visker, and J. A. van Arendonk. 2011. Genome-wide association of milk fatty acids in Dutch dairy cattle. BMC Genet. 12:43.

Bouwman, A. C., B. D. Valente, L. L. Janss, H. Bovenhuis, and G. J. Rosa. 2014. Exploring causal networks of bovine milk fatty acids in a multivariate mixed model context. Genet. Sel. Evol. 46:2.

Bouwman, A. C., M. H. Visker, J. A. van Arendonk, and H. Bovenhuis. 2012. Genomic regions associated with bovine milk fatty acids in both summer and winter milk samples. BMC Genet. 13:93.

Buchanan, F. C., A. G. Van Kessel, C. Waldner, D. A. Christensen, B. Laarveld, and S. M. Schmutz. 2003. Hot topic: an association between a leptin single nucleotide polymorphism and milk and protein yield. J. Dairy Sci. 86:3164-3166.

Buitenhuis, B., L. L. Janss, N. A. Poulsen, L. B. Larsen, M. K. Larsen, and P. Sørensen. 2014. Genome-wide association and biological pathway analysis for milk-fat composition in Danish Holstein and Danish Jersey cattle. BMC Genomics 15:1112.

Cecchinato, A., S. Chessa, C. Ribeca, C. Cipolat-Gotet, T. Bobbo, J. Casellas, and G. Bittante. 2015. Genetic variation and effects of candidate-gene polymorphisms on coagulation properties, curd firmness modeling and acidity in milk from Brown Swiss cows. Animal 9:1104-1112.

Cecchinato, A., C. Ribeca, S. Chessa, C. Cipolat-Gotet, F. Maretto, J. Casellas, and G. Bittante. 2014. Candidate gene association analysis for milk yield, composition, urea nitrogen and somatic cell scores in Brown Swiss cows. Animal 8:1062-1070.

Chessa, S., O. Bulgari, A. Rossoni, G. Ceriotti, and A. M. Caroli. 2013. Bovine $\beta$-casein: Detection of two single nucleotide polymorphisms by bidirectional allele specific polymerase chain reaction (BAS-PCR) and monitoring of their variation. OJAS 3:36-41.

Chilliard, Y., A. Ferlay, Y. Faulconnier, M. Bonnet, J. Rouel, and F. Bocquier. 2000. Adipose tissue metabolism and its role in adaptations to undernutrition in ruminants. Proc. Nutr. Soc. 59:127-134.

Cipolat-Gotet, C., A. Cecchinato, M. De Marchi, and G. Bittante. 2013. Factors affecting variation of different measures of cheese yield and milk nutrients recovery from an individual model cheesemanufacturing process. J. Dairy Sci. 96:7952-7965.

Clempson, A. M., G. E. Pollott, J. S. Brickell, N. E. Bourne, N. Munce, and D. C. Wathes. 2011. Evidence that leptin genotype is associated with fertility, growth, and milk production in Holstein cows. J. Dairy Sci. 94:3618-3628.

Cobanoglu, O., I. Zaitoun, Y. M. Chang, G. E. Shook, and H. Khatib. 2006. Effects of the signal transducer and activator of transcription 1 (STAT1) gene on milk production traits in Holstein dairy cattle. J. Dairy Sci. 89:4433-4437.

Conte, G., M. Mele, S. Chessa, B. Castiglioni, A. Serra, G. Pagnacco, and P. Secchiari. 2010. Diacylglycerol acyltransferase 1, stearoylCoA desaturase 1, and sterol regulatory element binding protein 1 gene polymorphisms and milk fatty acid composition in Italian Brown cattle. J. Dairy Sci. 93:753-763.

Dagnachew, B. S., and T. Ådnøy. 2014. Additive and dominance effects of casein haplotypes on milk composition and quality in Norwegian dairy goat. Small Rumin. Res. 122:59-69.

Dilzer, A., and Y. Park. 2012. Implication of conjugated linoleic acid (CLA) in human health. Crit. Rev. Food Sci. Nutr. 52:488-513. 
Falconer, D. S., and T. F. C. Mackay. 1996. Introduction to Quantitative Genetics. 4th ed. Longman, Essex, UK.

Feng, S., A. L. Lock, and P. C. Gasnsworthy. 2004. A rapid lipid separation method for determining fatty acid composition of milk. J. Dairy Sci. 87:3785-3788.

Feuermann, Y., S. J. Mabjeesh, and A. Shamay. 2004. Leptin affects prolactin action on milk protein and fat synthesis in the bovine mammary gland. J. Dairy Sci. 87:2941-2946.

Fievez, V., E. Colman, J. M. Castro-Montoya, I. Stefanov, and B. Vlaeminck. 2012. Milk odd- and branched-chain fatty acids as biomarkers of rumen function-An update. Anim. Feed Sci. Technol. 172:51-65

Gebauer, S. K., J. M. Chardigny, M. U. Jakobsen, B. Lamarche, A L. Lock, S. D. Proctor, and D. J. Baer. 2011. Effects of ruminant trans fatty acids on cardiovascular disease and cancer: A comprehensive review of epidemiological, clinical, and mechanistic studies. Adv. Nutr. 2:332-354.

German, J. B. 2011. Dietary lipids from an evolutionary perspective: sources, structures and functions. Matern. Child Nutr. 7(Suppl. 2):2-16.

Geweke, J. 1992. Evaluating the accuracy of sampling-based approaches to the calculation of posterior moments (with discussion). Pages 164-193 in Bayesian Statistics. J. O. Berger, J. M. Bernardo, A. P. Dawid, and A. F. M. Smith, ed. Oxford University Press, Oxford, UK.

Geyer, C. J. 1992. Practical Markov chain Monte Carlo. Stat. Sci. $7: 473-483$.

Krag, K., N. A. Poulsen, M. K. Larsen, L. B. Larsen, L. L. Janss, and B. Buitenhuis. 2013. Genetic parameters for milk fatty acids in Danish Holstein cattle based on SNP markers using a Bayesian approach. BMC Genet. 14:79.

Kramer, J. K. G., M. Hernandez, C. Cruz-Hernandez, J. Kraft, and M. E. R. Dugan. 2008. Combining results of two GC separations partly achieves determination of all cis and trans 16:1, 18:1, 18:2 and 18:3 except CLA isomers of milk fat as demonstrated using Ag-ion SPE fractionation. Lipids 43:259-273.

Kurokawa, N., E. H. Young, Y. Oka, H. Satoh, N. J. Wareham, M. S. Sandhu, and R. J. Loos. 2008. The ADRB3 Trp64Arg variant and BMI: A meta-analysis of 44833 individuals. Int. J. Obes. (Lond.) 32:1240-1249.

Li, C., D. Sun, S. Zhang, S. Wang, X. Wu, Q. Zhang, L. Liu, Y. Li, and L. Qiao. 2014. Genome wide association study identifies 20 novel promising genes associated with milk fatty acid traits in Chinese Holstein. PLoS ONE 9:e96186.

Marchitelli, C., G. Contarini, G. De Matteis, A. Crisà, L. Pariset, M. C. Scatà, G. Catillo, F. Napolitano, and B. Moioli. 2013. Milk fatty acid variability: Effect of some candidate genes involved in lipid synthesis. J. Dairy Res. 80:165-173.

Matsumoto, H., K. Sasaki, T. Bessho, E. Kobayashi, T. Abe, S. Sasazaki, K. Oyama, and H. Mannen. 2012. The SNPs in the ACACA gene are effective on fatty acid composition in Holstein milk. Mol. Biol. Rep. 39:8637-8644.

Mele, M. 2009. Designing milk fat to improve healthfulness and functional properties of dairy products: From feeding strategies to a genetic approach. Ital. J. Anim. Sci. 8:365-373.

Mele, M., G. Conte, B. Castiglioni, S. Chessa, N. P. Macciotta, A Serra, A. Buccioni, G. Pagnacco, and P. Secchiari. 2007. Stearoylcoenzyme A desaturase gene polymorphism and milk fatty acid composition in Italian Holsteins. J. Dairy Sci. 90:4458-4465.

Mele, M., A. Serra, A. Buccioni, G. Conte, A. Pollicardo, and P. Secchiari. 2008. Effect of soybean oil supplementation on milk fatty acid composition from Saanen goats fed diets with different forage:concentrate ratios. Ital. J. Anim. Sci. 7:297-311.

Mente, A., L. de Koning, H. S. Shannon, and S. S. Anand. 2009. A systematic review of the evidence supporting a causal link between dietary factors and coronary heart disease. Arch. Intern. Med. 169:659e69

Michal, J. J., Z. W. Zhang, and C. T. Gaskins. 2006. The bovine fatty acid binding protein 4 gene is significantly associated with marbling and subcutaneous fat depth in Wagyu $\times$ Limousin F2 crosses. Anim. Genet. 37:400-402.

Michas, G., R. Micha, and A. Zampelas. 2014. Dietary fats and cardiovascular disease: Putting together the pieces of a complicated puzzle. Atherosclerosis 234:320-328.

Muñoz, A., and M. Costa. 2013. Nutritionally mediated oxidative stress and inflammation. Oxid. Med. Cell. Longev. 2013:610950.

Nafikov, R. A., J. P. Schoonmaker, K.T. Korn, K. Noack, D. J. Garrick, K. J. Koehler, J. Minick-Bormann, J. M. Reecy, D. E. Spurlock, and D. C. Beitz. 2013a. Sterol regulatory element binding transcription factor 1 (SREBF1) polymorphism and milk fatty acid composition. J. Dairy Sci. 96:2605-2616.

Nafikov, R. A., J. P. Schoonmaker, K. T. Korn, K. Noack, D. J. Garrick, K. J. Koehler, J. Minick-Bormann, J. M. Reecy, D. E. Spurlock, and D. C. Beitz. 2013b. Association of polymorphisms in solute carrier family 27, isoform A6 (SLC27A6) and fatty acidbinding protein-3 and fatty acid-binding protein-4 (FABP3 and FABP4) with fatty acid composition of bovine milk. J. Dairy Sci. 96:6007-6021

Nafikov, R. A., J. P. Schoonmaker, K. T. Korn, K. Noack, D. J. Garrick, K. J. Koehler, J. Minick-Bormann, J. M. Reecy, D. E. Spurlock, and D. C. Beitz. 2014. Polymorphisms in lipogenic genes and milk fatty acid composition in Holstein dairy cattle. Genomics 104:572-581.

Pannier, L., A. M. Mullen, R. M. Hamill, P. C. Stapleton, T. Sweeney, and Z. Jiang. 2010. Association analysis of single nucleotide polymorphisms in DGAT1, TG and FABP4 genes and intramuscular fat in crossbred Bos taurus cattle. Meat Sci. 85:515-518.

Pegolo, S., A. Cecchinato, J. Casellas, G. Conte, M. Mele, S. Schiavon, and G. Bittante. 2016. Genetic and environmental relationships of detailed milk fatty acids profile determined by gas chromatography in Brown Swiss cows. J. Dairy Sci. 99:1315-1330.

Raftery, A. E., and S. M. Lewis. 1996. Implementing MCMC. Pages 115-130 in Markov Chain Monte Carlo in Practice. W. R. Gilks, D. J. Spiegelhalter, and S. Richardson, ed. Chapman and Hall, London, UK

Ramírez, O., R. Quintanilla, L. Varona, D. Gallardo, I. Diaz, R. N. Pena, and M. Amills. 2014. DECR1 and ME1 genotypes are associated with lipid composition traits in Duroc pigs. J. Anim. Breed. Genet. 131:46-52.

Rincon, G., A. Islas-Trejo, A. R. Castillo, D. E. Bauman, B. J. German, and J. F. Medrano. 2012. Polymorphisms in genes in the SREBP1 signalling pathway and SCD are associated with milk fatty acid composition in Holstein cattle. J. Dairy Res. 79:66-75.

Rudolph, M. C. T. D. Russell, P. Webb, M. C. Neville, and S. M. Anderson. 2011. Prolactin-mediated regulation of lipid biosynthesis genes in vivo in the lactating mammary epithelial cell. Am. J. Physiol. Endocrinol. Metab. 300:E1059-E1068.

Schennink, A., J. M. L. Heck, H. Bovenhuis, M. H. P. W. Visker, H. J. F. van Valenberg, and J. A. M. van Arendonk. 2008. Milk fatty acid unsaturation: Genetic parameters and effects of stearoyl-CoA desaturase (SCD1) and acyl CoA: Diacylglycerol acyltransferase 1 (DGAT1). J. Dairy Sci. 91:2135-2143.

Schennink, A., W. M. Stoop, M. H. Visker, J. M. Heck, H. Bovenhuis, J. J. van der Poel, H. J. van Valenberg, and J. A. van Arendonk. 2007. DGAT1 underlies large genetic variation in milk-fat composition of dairy cows. Anim. Genet. 38:467-473.

Schennink, A., W. M. Stoop, M. H. P. W. Visker, J. J. van der Poel, H. Bovenhuis, and J. A. M. van Arendonk. 2009. Short communication: Genome-wide scan for bovine milk-fat composition. II Quantitative trait loci for long-chain fatty acids. J. Dairy Sci. 92:4676-4682

Shingfield, K. J., M. Bonnet, and N. D. Scollan. 2013. Recent developments in altering the fatty acid composition of ruminant-derived foods. Animal 7:132-162.

Siri-Tarino, P.W., Q. Sun, F.B. Hu, and R.M. Krauss. 2010. Metaanalysis of prospective cohort studies evaluating the association of saturated fat with cardiovascular disease. Am. J. Clin. Nutr. 91:535e 46 
Sorensen, D., and D. Gianola. 2002. Likelihood, Bayesian, and MCMC Methods in Quantitative Genetics. Springer-Verlag, New York, NY.

Stoop, W. M., A. Schennink, M. H. Visker, E. Mullaart, J. A. van Arendonk, and H. Bovenhuis. 2009. Genome-wide scan for bovine milk-fat composition. I. Quantitative trait loci for short- and medium-chain fatty acids. J. Dairy Sci. 92:4664-4675.

Strillacci, M. G., E. Frigo, F. Canavesi, Y. Ungar, F. Schiavini, L. Zaniboni, L. Reghenzani, M. C. Cozzi, A. B. Samoré, Y. Kashi, E. Shimoni, R. Tal-Stein, M. Soller, E. Lipkin, and A. Bagnato. 2014. Quantitative trait loci mapping for conjugated linoleic acid, vaccenic acid and $\Delta(9)$-desaturase in Italian Brown Swiss dairy cattle using selective DNA pooling. Anim. Genet. 45:485-499.

Sumner-Thomson, J. M., J. L. Vierck, and J. P. McNamara. 2011. Differential expression of genes in adipose tissue of first-lactation dairy cattle. J. Dairy Sci. 94:361-369.
Wang, X., C. Maltecca, R. Tal-Stein, E. Lipkin, and H. Khatib. 2008. Association of bovine fibroblast growth factor 2 (FGF2) gene with milk fat and productive life: An example of the ability of the candidate pathway strategy to identify quantitative trait genes. J. Dairy Sci. 91:2475-2480.

Welte, T., K. Garimorth, S. Philipp, and W. Doppler. 1994. Prolactin-dependent activation of a tyrosine phosphorylated DNA binding factor in mouse mammary epithelial cells. Mol. Endocrinol. 8:1091-1102.

Zetouni, L., G. M. de Camargo, P. D. da Silva Fonseca, F. M. Gil, N. A. Lugo, R. R. Aspilcueta-Borquis, M. Cervini, and H. Tonhati. 2013. Effects of a single nucleotide polymorphism in the leptin gene on the productive traits of dairy buffaloes (Bubalus bubalis) Mol. Biol. Rep. 40:5159-5163. 